\title{
Assessment of mucocele formation after endoscopic nasoseptal flap reconstruction of skull base defects
}

\author{
Qasim Husain, B.S., ${ }^{1}$ Saurin Sanghvi, B.S., ${ }^{1}$ Olga Kovalerchik, B.A., ${ }^{1}$ Pratik A. Shukla, M.D., ${ }^{1}$ \\ Osamah J. Choudhry, M.D., ${ }^{2}$ James K. Liu, M.D., ${ }^{1,2,3}$ and Jean Anderson Eloy, M.D., F.A.C.S. ${ }^{1,2,3}$
}

\begin{abstract}
Advances in endoscopic skull base (SB) surgery have led to the resection of increasingly larger cranial base lesions, resulting in large SB defects. These defects have initially led to increased postoperative cerebrospinal fluid (CSF) leaks. The development of the vascularized pedicled nasoseptal flap (PNSF) has successfully reduced postoperative CSF leaks. Mucocele formation, however, has been reported as a complication of this technique. In this study, we analyze the incidence of mucocele formation after repair of SB defects using a PNSF. A retrospective review was performed from December 2008 to December 2011 to identify patients who underwent PNSF reconstruction for large ventral SB defects. Demographic data, defect site, incidence of postoperative CSF leaks, and rate of mucocele formation were collected. Seventy patients undergoing PNSF repair of SB defects were identified. No postoperative mucocele formation was noted at an average radiological follow-up of 11.7 months (range, 3-36.9 months) and clinical follow-up of 13.8 months (range, 3-38.9 months), making the overall mucocele rate 0\%. The postoperative CSF leak rate was 2.9\%. Proper closure of SB defects is crucial to prevent CSF leaks. The PNSF is an efficient technique for these repairs. Although this flap may carry an inherent risk of mucocele formation when placed over mucosalized bone during repair, we found that meticulous and strategic removal of mucosa from the site of flap placement resulted in a $0 \%$ incidence of postoperative mucocele formation in our cohort.
\end{abstract}

(Allergy Rhinol 4:e27-e31, 2013; doi: 10.2500/ar.2013.4.0050)

$\mathbf{W}^{\prime \prime}$ ith the advent of advanced endoscopic techniques, there has been an upsurge in the minimally invasive approaches for resection of ventral skull base (SB) tumors. ${ }^{1-8}$ Although highly effective, these procedures often result in large SB defects that can lead to high-flow cerebrospinal fluid (CSF) leaks. These defects typically necessitate meticulous multilayer reconstruction to prevent postoperative failure. The vascularized pedicled nasoseptal flap (PNSF) used in a multilayered fashion is currently the workhorse for closure of these high-flow CSF leaks in many major academic centers, and has been shown to decrease postoperative CSF leaks. ${ }^{9-24}$

A potential risk after reconstruction of SB defects is mucocele formation. ${ }^{25-28}$ A sinonasal mucocele is a mucus-containing sac lined with epithelium that forms secondary to obstructed sinus drainage after previous

From the Departments of ${ }^{1}$ Otolaryngology-Head and Neck Surgery and ${ }^{2}$ Neurological Surgery and ${ }^{3}$ Center for Skull Base and Pituitary Surgery, Neurological Institute of New Jersey, University of Medicine and Dentistry of New Jersey-New Jersey Medical School, Newark, New Jersey

Presented at the 58th annual meeting of the American Rhinologic Society, Washington, D.C., September 8, 2012

The authors have no conflicts of interest to declare pertaining to this article Address correspondence and reprint requests to Jean Anderson Eloy, M.D., F.A.C.S. Department of Otolaryngology-Head and Neck Surgery, University of Medicine and Dentistry of New Jersey -New Jersey Medical School, 90 Bergen Street, Suite 8100, Newark, NJ 07103

E-mail address: jean.anderson.eloy@gmail.com

Published online May 14, 2013

Copyright (C) 2013, OceanSide Publications, Inc., U.S.A. sinus surgeries, posttraumatic lesions, chronic sinusitis, tumors, and infectious and inflammatory processes. ${ }^{29}$ Although benign, mucoceles may produce a growing mass with the potential to erode bone and adjacent orbital and neural structures, making their removal imperative. ${ }^{30}$

Postoperative mucocele formation after endoscopic $\mathrm{SB}$ reconstruction has previously been reported. ${ }^{35,26}$ Bleier et al..$^{31}$ reported a $3.6 \%$ mucocele rate after SB defect repair using the vascularized PNSF. Vaezeafshar et $a .^{32}$ also reported a mucocele 4 months after SB reconstruction with a vascularized PNSF. Although the exact pathogenesis of the mucocele remains controversial, techniques to decrease the incidence of postoperative mucocele formation after SB defect repairs have been described. ${ }^{27,33}$ The fundamental principle in preventing postoperative mucocele formation after PNSF reconstruction of SB defects involves denuding of the mucosa at the site of flap inset. ${ }^{14,15,28,34}$ In this study we evaluate our experience with judicious demucosalization of the site of flap placement to prevent postoperative mucocele formation in 70 patients who underwent PNSF reconstruction of large ventral SB defects.

\section{MATERIALS AND METHODS}

\section{Experimental Design}

The protocol for this study was reviewed and approved by the Institutional Review Board of the University of Medicine and Dentistry of New Jersey-New 
Jersey Medical School, Newark, NJ. A retrospective chart review was performed to identify patients undergoing endoscopic endonasal reconstruction of large ventral SB defects from December 2008 to December 2011. Demographic data, diagnosis, surgical procedure, rate of mucocele formation, and postoperative CSF leak were collected. Exclusion criteria included patients with $<3$ months (90 days) of radiological and clinical follow-up, patients with spontaneous low-flow CSF leaks because their defect sizes did not need PNSF for closure, and patients who underwent combined cranionasal approaches. Therefore, all patients in this study had high-flow CSF leak. For sellar defects, only grade 3, large diaphragmatic/dural defects were included in this analysis. ${ }^{35}$

\section{Surgical Technique}

The PNSF is harvested at the beginning of the operation when we anticipate a high-flow CSF leak (as for intradural lesions). We prepare the site of repair by denuding $\sim 1 \mathrm{~cm}$ of mucosa around the defect site. If a lateral sphenoid recess, sellar, or tuberculum sellae/planum sphenoidale repair is planned, the sphenoid sinus is also demucosalized. In cases of a deep lateral sphenoid recess, angled endoscopes and curved instruments are used to access this area for demucosalization after extended sphenoid sinusotomies. The area under the pedicle is also denuded of any mucosa. In cases of cribriform and other defects distant from the pedicle vascular supply site (sphenopalatine foramen), the area of SB along the course of the pedicle is also demucosalized to prevent any trapping of mucosa under the entire course of the PNSF. The CSF leak is then repaired in a multilayer fashion by initially reducing a high-flow CSF leak to a low-flow leak and rotating the PNSF into place as the last layer of the repair. The PNSF is meticulously positioned over the SB defect, and a single layer of Surgicel (Ethicon, Somerville, NJ) is then placed over the flap margin to improve bone apposition. In some instances (early in our series) a dural sealant was placed over the PNSF, which is subsequently bolstered in place by several large units (1-2 cm in diameter) of Gelfoam (Pharmacia, Kalamazoo, Michigan) soaked in gentamicin. We have stopped using dural sealants since we found no benefits to their use in a recent retrospective analysis. ${ }^{21}$ A Merocel nasal tampon (Medtronic Xomed, Jacksonville, FL) covered with bacitracin is then used to further support the closure (Fig. 1).

\section{Postoperative Management}

Routine postsurgical head and sinus computed tomography is obtained within 24 hours. Magnetic resonance imaging of the brain and sinuses is performed within 48 hours of surgery. Patients are started and kept on a $\beta$-lactamase-inhibiting penicillin or third-generation cephalosporin until removal of the nasal packing $\sim 10$ days after surgery. Postoperative CSF leak precautions and stool softeners are initiated. Outpatient follow-up was performed using rigid nasal endoscopy for conservative and careful debridement and flap surveillance.

\section{RESULTS}

Seventy patients undergoing PNSF reconstruction of ventral SB defects are included in this analysis. The mean age was 47.9 years (range, 14-81 years). There were $37.1 \%$ men and $62.9 \%$ women in this study. Average radiological and clinical follow-up was 11.7 months (range, 3-36.9 months) and 13.8 months (range, 3-38.9 months), respectively. Defect sites included sellar (34), cribriform (15), tuberculum sellae/planum sphenoidale (18), lateral sphenoid recess (2), and clival (1). Postoperative CSF leak rate was $2.9 \%$. No mucoceles were encountered in any patients in this study at latest follow-up, leading to a $0 \%$ mucocele formation rate.

\section{DISCUSSION}

Advancements in surgical technology have led to minimally invasive routes for access and resection of ventral SB lesions. Despite the improved access for tumor resection allowed through these approaches, the resulting large SB defects create the potential for postoperative CSF leaks. The creation of the PNSF has significantly improved the rate of postoperative CSF leakage after large SB defect repairs. ${ }^{36}$ However, the use of the PNSF has been associated with postoperative mucocele formation. ${ }^{31,32}$

Mucocele formation can appear as a late consequence of SB defect reconstruction. These slow-growing, benign lesions are most often found in the frontal and ethmoid sinuses. ${ }^{30}$ The frontal sinus is the most common location, because the nasofrontal outflow pathway can become obstructed by polyps, bony tumors, adhesions from prior surgery, sinusitis, trauma, or anatomic/congenital variation. ${ }^{37}$ The mechanism underlying the formation of a mucocele involves the gradual accumulation of mucous material in an obstructed sinus leading to compromised ventilation. ${ }^{26}$ The enlarging mass produces a progressive distension of the bony walls until there is compression of orbital and neural structures. ${ }^{30}$ Such patients typically present with headache, proptosis, diplopia, nasal congestion, and fluid leakage. ${ }^{38-40}$ Although mucocele formation is considered a late complication, its timing may be variable depending on the underlying etiology. Postoperative mucocele formation after endoscopic sinus surgery has been reported with average time lapse between 2 and 6 years. ${ }^{26,39,41-43}$ Nonetheless, prior reports of mucocele formation after vascularized PNSF repair have been reported at 46 days $^{31}$ and at 4 months. ${ }^{32}$

Different ideologies exist for the pathogenesis of mucocele formation, because there are a variety of etiologies that lead to sinus obstruction. For traumatic cases 

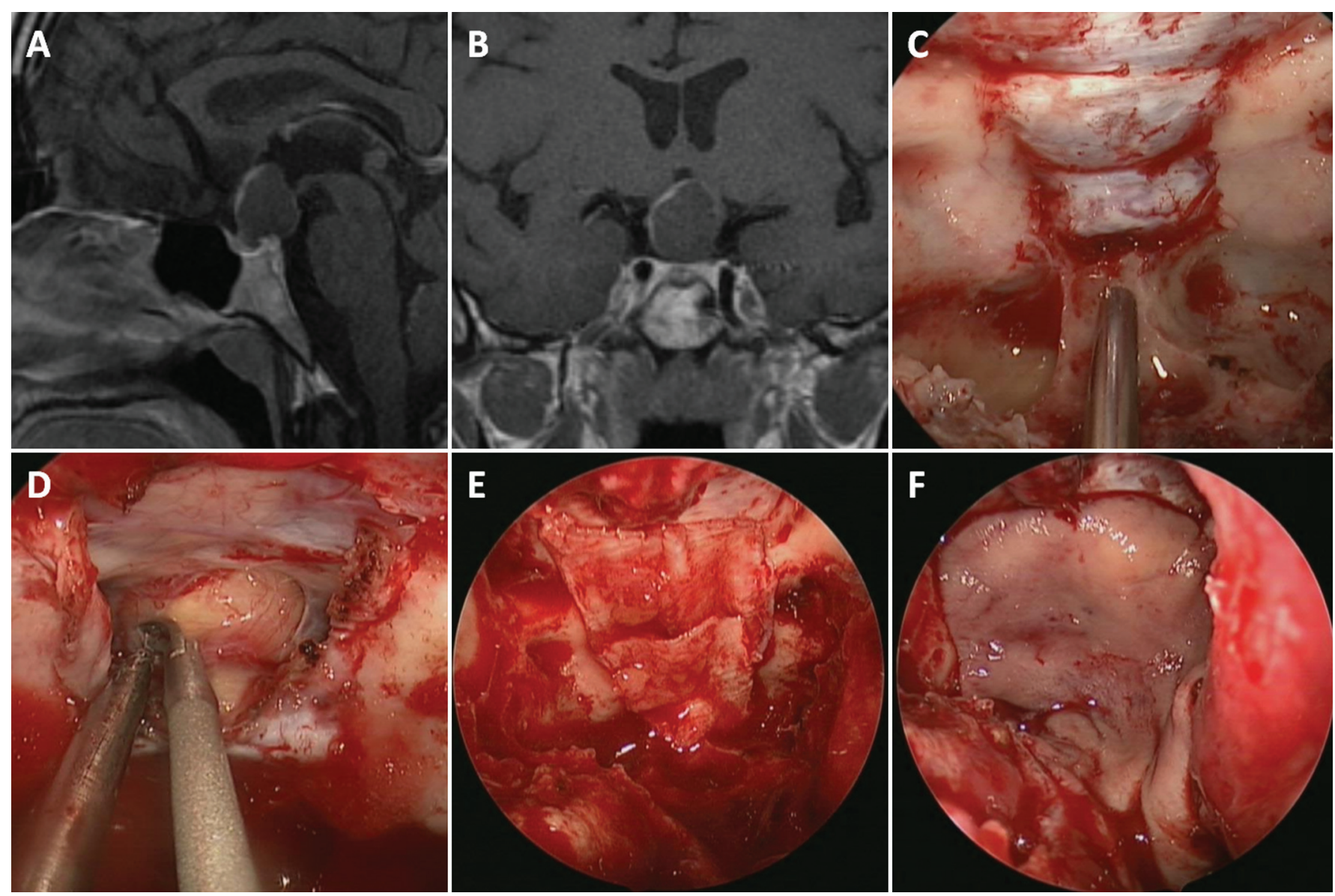

Figure 1. T1-weighted gadolinium-enhanced (A) sagittal and (B) coronal magnetic resonance imaging (MRI) of a patient with a suprasellar retrochiasmatic skull base (SB) tumor resected via an endoscopic transplanum transtuberculum approach. (C) Intraoperative view after bone removal. Please note the removal of the sphenoid sinus mucosa. (D) Intraoperative view after dural and arachnoid opening depicting early tumor dissection. (E) Stepwise repair of the SB dural defect with initial conversion of high-flow to low-flow cerebral spinal fluid (CSF) leak using autologous fascia lata placed over the transplanum SB defect; and (F) rotation and positioning of pedicled nasoseptal flap (PNSF) over the defect.

it has been postulated that the implantation of respiratory epithelium after fracture or iatrogenic exposure is responsible for the formation of these mucoceles. ${ }^{29}$ Lund et al. ${ }^{44}$ suggested the role of inflammation in the pathophysiology of mucocele enlargement by indicating that the release of proinflammatory cytokines from the mucocele wall can result in osteoclastic bone resorption and destruction of adjacent tissue. ${ }^{44,45}$ Endoscopic endonasal surgery increases the risk of nasofrontal outflow pathway stenosis and subsequent mucocele formation as evidenced by the clear increase in incidence of mucocele formation since the 1990s paralleling the increasing number of sinus surgery procedures. ${ }^{41,43}$ Inaccurate patch grafting between bone and mucosa during closure of SB defects is thought to be another iatrogenic cause of mucocele formation. ${ }^{27,28}$

Postsurgically, entrapment of mucosa, meatal adhesions, and improper flap placement have been reported as potential causes for mucocele formation. ${ }^{27,28,41} \mathrm{In}$ light of these risks, endoscopic SB surgeons have used different techniques to reduce the incidence of postop- erative mucocele formation. Denuding the sinonasal mucosa adjacent to the SB defect has been reported as a means for reducing this risk. ${ }^{14,15,27,34}$ Entrapped mucosal glands aberrantly produce mucous that increases the chances of eventual mucocele formation. ${ }^{27,46} \mathrm{In}$ addition, avoiding trauma of the healthy mucosa surrounding the middle meatus and extensive cleansing of the ethmoid cavity might decrease the risk of adhesions and development of mucocele. ${ }^{26}$ Another recommendation is to avoid the overlapping of flaps when using bilateral PNSF, because it may predispose to mucocele formation due to the mucosal entrapment mechanism. ${ }^{34}$

Verilaud et al. ${ }^{26}$ evaluated the formation of mucoceles after endoscopic repair of CSF fistulas in children with autologous free grafts, most commonly with the middle turbinate. Mucocele development occurred in $50 \%$ of their cases in their 12 patient series suggesting that the frequency may be higher in the pediatric population. This idea of increased mucocele formation in the pediatric population is consistent with McLaughlin et al., ${ }^{47}$ who 
attributed this rise to the fontal sinus, which does not fully form until age 19 years. Di Rocco et al. ${ }^{25}$ showed that in 28 pediatric patients undergoing repair of SB defects, $14 \%$ developed mucocele at a mean of 26.7 months follow-up. Both studies used bony and mucosal fragments of the middle turbinate, as well as cartilage from the nasal septum and auricle. ${ }^{25}$

Other authors have commented on mucocele formation after SB reconstruction. Bleier et al. ${ }^{31}$ discussed their results of 28 patients undergoing SB reconstruction with a vascularized PNSF. Partial removal of the sinus mucosa surrounding the cranial base defect was used in the procedure resulting in a $3.6 \%$ incidence of postoperative mucocele formation ( 1 out of 28 , with the lone mucocele detected at postoperative day 46). The authors used partial mucosal denuding to prevent adjacent neurovascular injury, prolonged postoperative crust formation, and increased scar formation. ${ }^{31}$ Vaezeafshar et $a .^{32}$ reported on a case of sphenoid sinus mucocele under a vascularized PNSF detected 4 months postoperatively. Nyquist et al. ${ }^{34}$ reported no mucocele formation in five patients who underwent $\mathrm{SB}$ reconstruction using bilateral PNSFs.

In our series, we judiciously denude $1 \mathrm{~cm}$ of mucosa surrounding the ASB defect before PNSF placement. We also denude the sphenoid sinus during repair of large lateral sphenoid recess defects, large sellar defects, and all tuberculum sellae/planum sphenoidale defects. This technique resulted in a $0 \%$ incidence of mucocele formation at latest follow-up in our series of 70 patients who underwent ventral SB defect repair and a $2.9 \%$ postoperative CSF leak rate. Reported mucocele rates in previous studies after endoscopic endonasal SB surgery in the pediatric literature was estimated to be at 50 and $14 \% .{ }^{25,26}$ However, these studies did not use the PNSF, but instead, used autologous free flaps from the middle turbinate to reconstruct the defect. ${ }^{25,26}$ It may be important to note that the indication for surgery in this population was for posttraumatic and congenital defects. Therefore, it is possible that the etiology of the defect as well as the pediatric population may have increased susceptibility to mucocele formation.

We believe that the prevention of mucocele is facilitated by meticulous care of the mucosa surrounding the cranial base defect. Judicious removal of the mucosa surrounding the SB defect and meticulous placement of the PNSF are techniques we use to reduce the incidence of mucocele formation. ${ }^{26-28,34}$ This may in part explain the $0 \%$ incidence of mucocele formation in this cohort.

Although these results are quite promising and showed a $0 \%$ incidence of postoperative mucocele formation, there are several limitations to this study. Because mucocele formation can occur as a late complication, our relatively short follow-up period should be acknowledged. Nonetheless, mucocele formation has been reported as early as 46 days and 4 months after PNSF repair of SB defects, making our findings acceptable. Mucoceles are slow-growing processes that often are diagnosed incidentally and may be present for years before becoming symptomatic. Consequently, our current radiographic follow-up, which is considerably longer than the existing literature examining mucocele formation after PNSF repair of SB defects, is reasonable. Additionally, this study is subject to the limitations found in all retrospective studies and can benefit from validation through multi-institutional efforts as well as prospective randomized controlled double-blind analyses.

\section{CONCLUSION}

Successful endoscopic reconstruction of large ventral SB defects is frequently performed with the vascularized PNSF. Although previous studies have reported postoperative mucocele formation with this technique, we found a $0 \%$ incidence of this complication in our cohort. Judicious mucosal denuding around the SB defect and meticulous closure can minimize this potential risk of mucocele formation after PNSF repair of ventral SB defects.

\section{REFERENCES}

1. Liu JK, Christiano LD, Patel SK, et al. Surgical nuances for removal of olfactory groove meningiomas using the endoscopic endonasal transcribriform approach. Neurosurg Focus 30:E3, 2011.

2. Liu JK, Christiano LD, Patel SK, and Eloy JA. Surgical nuances for removal of retrochiasmatic craniopharyngioma via the endoscopic endonasal extended transsphenoidal transplanum transtuberculum approach. Neurosurg Focus 30:E14, 2011.

3. Liu JK, Christiano LD, Patel SK, et al. Surgical nuances for removal of tuberculum sellae meningiomas with optic canal involvement using the endoscopic endonasal extended transsphenoidal transplanum transtuberculum approach. Neurosurg Focus 30:E2, 2011.

4. Patel AA, Friedel ME, Liu JK, and Eloy JA. Endoscopic endonasal resection of extensive anterior skull base sinonasal osteoblastoma. Otolaryngol Head Neck Surg 147:594-596, 2012.

5. Liu JK, and Eloy JA. Endoscopic endonasal transplanum transtuberculum approach for resection of retrochiasmatic craniopharyngioma. J Neurosurg 32(suppl):E2, 2012.

6. Tessema B, Eloy JA, Folbe AJ, et al. Endoscopic management of sinonasal hemangiopericytoma. Otolaryngol Head Neck Surg 146:483-486, 2012.

7. Liu JK, and Eloy JA. Expanded endoscopic endonasal transcribriform approach for resection of anterior skull base olfactory schwannoma. J Neurosurg 32(suppl):E3, 2012.

8. Husain Q, Patel SK, Soni RS, et al. Celebrating the golden anniversary of anterior skull base surgery: Reflections on the past 50 years and its historical evolution. Laryngoscope 123:6472, 2013.

9. Eloy JA, Patel AA, Shukla PA, et al. Early harvesting of the vascularized pedicled nasoseptal flap during endoscopic skull base surgery. Am J Otolaryngol 2013 Jan 16, DOI: 10.1016/ j.amjoto. 2012.10.005 [Epub ahead of print]. 
10. Eloy JA, Kalyoussef E, Choudhry OJ, et al. Salvage endoscopic nasoseptal flap repair of persistent cerebrospinal fluid leak after open skull base surgery. Am J Otolaryngol 33:735-740, 2012.

11. Eloy JA, Patel SK, Shukla PA, et al. Triple-layer reconstruction technique for large cribriform defects after endoscopic endonasal resection of anterior skull base tumors. Int Forum Allergy Rhinol 3:204-211, 2013.

12. Kassam AB, Prevedello DM, Carrau RL, et al. Endoscopic endonasal skull base surgery: Analysis of complications in the authors' initial 800 patients. J Neurosurg 114:1544-1568, 2011.

13. Shah RN, Surowitz JB, Patel MR, et al. Endoscopic pedicled nasoseptal flap reconstruction for pediatric skull base defects. Laryngoscope 119:1067-1075, 2009.

14. Hadad G, Bassagasteguy L, Carrau RL, et al. A novel reconstructive technique after endoscopic expanded endonasal approaches: Vascular pedicle nasoseptal flap. Laryngoscope 116: 1882-1886, 2006.

15. Kassam AB, Thomas A, Carrau RL, et al. Endoscopic reconstruction of the cranial base using a pedicled nasoseptal flap. Neurosurgery 63:ONS44-52, 2008.

16. Zanation AM, Carrau RL, Snyderman CH, et al. Nasoseptal flap reconstruction of high flow intraoperative cerebral spinal fluid leaks during endoscopic skull base surgery. Am J Rhinol Allergy 23:518-521, 2009.

17. Zanation AM, Carrau RL, Snyderman $\mathrm{CH}$, et al. Nasoseptal flap takedown and reuse in revision endoscopic skull base reconstruction. Laryngoscope 121:42-46, 2011.

18. Snyderman $\mathrm{CH}$, Kassam AB, Carrau R, and Mintz A. Endoscopic reconstruction of cranial base defects following endonasal skull base surgery. Skull Base 17:73-78, 2007.

19. Liu JK, Schmidt RF, Choudhry OJ, et al. Surgical nuances for nasoseptal flap reconstruction of cranial base defects with highflow cerebrospinal fluid leaks after endoscopic skull base surgery. Neurosurg Focus 32:E7, 2012.

20. Schmidt RF, Choudhry OJ, Raviv J, et al. Surgical nuances for the endoscopic endonasal transpterygoid approach to lateral sphenoid sinus encephaloceles. Neurosurg Focus 32:E5, 2012.

21. Eloy JA, Choudhry OJ, Friedel ME, et al. Endoscopic nasoseptal flap repair of skull base defects: Is addition of a dural sealant necessary? Otolaryngol Head Neck Surg 147:161-166, 2012.

22. Eloy JA, Choudhry OJ, Shukla PA, et al. Nasoseptal flap repair after endoscopic transsellar versus expanded endonasal approaches: Is there an increased risk of postoperative cerebrospinal fluid leak? Laryngoscope 122:1219-1225, 2012.

23. Eloy JA, Kuperan AB, Choudhry OJ, et al. Efficacy of the pedicled nasoseptal flap without cerebrospinal fluid (CSF) diversion for repair of skull base defects: Incidence of postoperative CSF leaks. Int Forum Allergy Rhinol 2:397-401, 2012.

24. Eloy JA, Choudhry OJ, Christiano LD, et al. Double flap technique for reconstruction of anterior skull base defects after craniofacial tumor resection: Technical note. Int Forum Allergy Rhinol 2012 Oct 4. DOI: 10.1002/alr.21092 [Epub ahead of print].

25. Di Rocco F, Couloigner V, Dastoli P, et al. Treatment of anterior skull base defects by a transnasal endoscopic approach in children. J Neurosurg Pediatr 6:459-463, 2010.

26. Verillaud B, Genty E, Leboulanger N, et al. Mucocele after transnasal endoscopic repair of traumatic anterior skull base fistula in children. Int J Pediatr Otorhinolaryngol 75:1137-1142, 2011.

27. Eloy JA, Fatterpekar GM, Bederson JB, and Shohet MR. Intracranial mucocele: An unusual complication of cerebrospinal fluid leakage repair with middle turbinate mucosal graft. Otolaryngol Head Neck Surg 137:350-352, 2007.
28. Wang L, Kim J, and Heilman CB. Intracranial mucocele as a complication of endoscopic repair of cerebrospinal fluid rhinorrhea: Case report. Neurosurgery 45:1243-1245, 1999.

29. Pero CD, and Nuss DW. Transnasal endoscopic removal of orbital, ethmoid sinus, and anterior skull base foreign body with mucocele formation. Skull Base 18:417-422, 2008.

30. Delfini R, Missori P, Iannetti G, et al. Mucoceles of the paranasal sinuses with intracranial and intraorbital extension: Report of 28 cases. Neurosurgery 32:901-906, 1993.

31. Bleier BS, Wang EW, Vandergrift WA II, and Schlosser RJ. Mucocele rate after endoscopic skull base reconstruction using vascularized pedicled flaps. Am J Rhinol Allergy 25:186-187, 2011.

32. Vaezeafshar R, Hwang PH, Harsh G, and Turner JH. Mucocele formation under pedicled nasoseptal flap. Am J Otolaryngol 33:634-636, 2012.

33. Devars du Mayne M, Moya-Plana A, Malinvaud D, et al. Sinus mucocele: Natural history and long-term recurrence rate. Eur Ann Otorhinolaryngol Head Neck Dis 129:125-130, 2012.

34. Nyquist GG, Anand VK, Singh A, and Schwartz TH. Janus flap: Bilateral nasoseptal flaps for anterior skull base reconstruction. Otolaryngol Head Neck Surg 142:327-331, 2010.

35. Esposito F, Dusick JR, Fatemi N, and Kelly DF. Graded repair of cranial base defects and cerebrospinal fluid leaks in transsphenoidal surgery. Neurosurgery 60:295-303, 2007.

36. Harvey RJ, Parmar P, Sacks R, and Zanation AM. Endoscopic skull base reconstruction of large dural defects: A systematic review of published evidence. Laryngoscope 122:452-459, 2012.

37. Plikaitis CM, Purzycki AR, Couture D, and David LR. Pediatric frontal mucocele secondary to a bifid frontal sinus septum. J Craniofac Surg 21:1525-1528, 2010.

38. Constantinidis J, Steinhart H, Schwerdtfeger K, et al. Therapy of invasive mucoceles of the frontal sinus. Rhinology 39:33-38, 2001.

39. Koudstaal MJ, van der Wal KG, Bijvoet HW, et al. Post-trauma mucocele formation in the frontal sinus; a rationale of followup. Int J Oral Maxillofac Surg 33:751-754, 2004.

40. Smoot EC III, Bowen DG, Lappert P, and Ruiz JA. Delayed development of an ectopic frontal sinus mucocele after pediatric cranial trauma. J Craniofac Surg 6:327-331, 1995.

41. Busaba NY, and Salman SD. Ethmoid mucocele as a late complication of endoscopic ethmoidectomy. Otolaryngol Head Neck Surg 128:517-522, 2003.

42. Mourouzis C, Evans BT, and Shenouda E. Late presentation of a mucocele of the frontal sinus: 50 Years postinjury. J Oral Maxillofac Surg 66:1510-1513, 2008.

43. Raynal M, Peynegre R, Beautru R, and Coste A. Sinus mucoceles and surgery in iatrogenic diseases. Ann Otolaryngol Chir Cervicofac 116:85-91, 1999.

44. Lund VJ, Henderson B, and Song Y. Involvement of cytokines and vascular adhesion receptors in the pathology of frontoethmoidal mucocoeles. Acta Otolaryngol 113:540-546, 1993.

45. Herman P, Lot G, Guichard JP, et al. Mucocele of the sphenoid sinus: A late complication of transsphenoidal pituitary surgery. Ann Otol Rhinol Laryngol 107:765-768, 1998.

46. Hilding A. Experimental surgery of the nose and sinuses, III: Results following partial and complete removal of the lining mucous membrane from the frontal sinus of the dog. Arch Otolaryngol Head Neck Surg 17:760-778, 1933.

47. McLaughlin RB Jr, Rehl RM, and Lanza DC. Clinically relevant frontal sinus anatomy and physiology. Otolaryngol Clin North Am 34:1-22, 2001. 